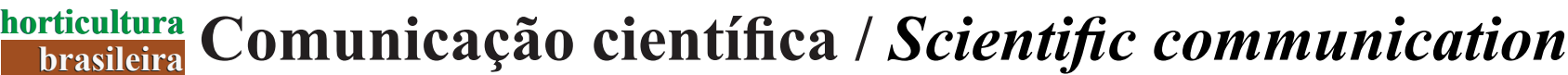

KURTZ, C; PAULETTI, V; FAYAD, JA; VIEIRA NETO, J. 2016. Crescimento e absorção de nutrientes pela cultivar de cebola Bola Precoce. Horticultura Brasileira 34: 279-288. DOI - http://dx.doi.org/10.1590/S0102-053620160000200020

\section{Crescimento e absorção de nutrientes pela cultivar de cebola Bola Precoce}

\author{
Claudinei Kurtz ${ }^{1,2}$; Volnei Pauletti'; Jamil A Fayad ${ }^{3}$; João Vieira Neto \\ 'Universidade Federal do Paraná (UFPR), Curitiba-PR, Brasil; kurtz@epagri.com.br; vpauletti@ufpr.com.br; ${ }^{2}$ Empresa de Pesquisa \\ Agropecuária e Extensão Rural de Santa Catarina (EPAGRI), Ituporanga-SC, Brasil; joaoneto@epagri.com.br; ${ }^{3}$ EPAGRI, Florianópolis- \\ SC,Brasil; jamil@epagri.sc.gov.br
}

\section{RESUMO}

No Sul, principal região produtora de cebola do Brasil, não se dispõe, atualmente, de pesquisas sobre a dinâmica de absorção de nutrientes, exigência nutricional e exportação de nutrientes para o sistema de transplante de mudas, que abrange mais de $70 \%$ da área cultivada com esta hortaliça. Objetivou-se determinar o crescimento, acúmulo, partição e as taxas de absorção de nutrientes para a cultivar de cebola Bola Precoce no sistema de transplante na região Sul do Brasil. O presente trabalho foi conduzido a campo na Estação Experimental da Epagri, município de Ituporanga-SC. O delineamento experimental foi em blocos casualizados com 18 tratamentos e cinco repetições. Cada tratamento correspondeu a uma época de avaliação/ coleta realizada em intervalos de sete dias. Cada parcela foi constituída de 27 plantas e para cada avaliação foram coletadas as cinco plantas centrais de cada parcela. Foi avaliado o acúmulo de massa seca, taxa de crescimento absoluto, teor e conteúdo dos nutrientes $\mathrm{N}, \mathrm{P}, \mathrm{K}, \mathrm{Ca}, \mathrm{Mg}, \mathrm{Zn}, \mathrm{Fe}, \mathrm{Cu}, \mathrm{B}$ e Mn nas folhas e bulbos e determinadas as taxas de absorção. $\mathrm{O}$ crescimento foi lento e a demanda por nutrientes baixa na primeira metade do ciclo até aos 60 dias após o transplante (DAT). Durante o período de bulbificação (61-119 DAT), o crescimento e a absorção de nutrientes foram intensificados e a planta acumulou $84 \%$ da MS total e entre 73 e $89 \%$ do total de nutrientes. No final do ciclo, para uma produtividade média de $37,34 \mathrm{t} / \mathrm{ha}$, a sequência de acúmulo de nutrientes verificada em ordem decrescente foi $\mathrm{N}>\mathrm{K}>\mathrm{Ca}>\mathrm{P}>\mathrm{Mg}>\mathrm{Fe}>\mathrm{B}>\mathrm{Mn}>\mathrm{Zn}>\mathrm{Cu}$, com 101,4; 86,5; 46,6; 34,$5 ; 12,1 \mathrm{~kg} / \mathrm{ha}$ e $761 ; 221 ; 150 ; 84$ e $34 \mathrm{~g} / \mathrm{ha}$, respectivamente. Dessa quantidade, 57,$5 ; 52,8 ; 42,3 ; 68,5 ; 57,3 ; 37,6 ; 70,7 ; 41,9 ; 73,6$ e $71,6 \%$, respectivamente, foram depositados no bulbo.

Palavras-chave: Allium cepa, nutrição de plantas, macronutrientes, micronutrientes.

\begin{abstract}
Growth and absorption of nutrients by onion cultivar Bola Precoce

In Southern Brazil, the Country main onion-producing region, research is not available on the dynamics of nutrient absorption, nutritional requirement and exportation of nutrients for the seedling transplanting system, which covers over $70 \%$ of the cultivated area with this vegetable crop. The objective was to determine growth, accumulation, partitioning and nutrient absorption rates of onion cultivar Bola Precoce in the transplanting system in the Southern Brazil. This study was carried out under field conditions at the Experimental Station of Epagri, municipality of Ituporanga, Santa Catarina State, Brazil. The experimental design was randomized blocks with 18 treatments and five replications. Each treatment corresponded to a time of assessment/collect held at intervals of seven days. Each plot consisted of 27 plants and for each evaluation we collected the five central plants in each plot. It was evaluated the dry matter accumulation, absolute growth rate, content of nutrients $\mathrm{N}, \mathrm{P}, \mathrm{K}, \mathrm{Ca}, \mathrm{Mg}, \mathrm{Zn}, \mathrm{Fe}, \mathrm{Cu}, \mathrm{B}$ and $\mathrm{Mn}$ in the leaves and bulbs and determined absorption rates. Growth was slow and demand for nutrients low in the first half of the cycle up to 60 days after transplanting (DAT). During bulbification (61-119 DAT), growth and nutrient absorption were enhanced and plants accumulated $84 \%$ of the total dry matter and from 73 to $89 \%$ of the nutrients. At the end of the cycle, for an average yield of $37.34 \mathrm{t} / \mathrm{ha}$, nutrient accumulation in descending order was $\mathrm{N}>\mathrm{K}>\mathrm{Ca}>\mathrm{P}>\mathrm{Mg}>\mathrm{Fe}>\mathrm{B}>\mathrm{Mn}>\mathrm{Zn}>\mathrm{Cu}$, with $101.4 ; 86.5 ; 46.6 ; 34.5 ; 12.1 \mathrm{~kg} / \mathrm{ha}$ and $761 ; 221 ; 150 ; 84$ and $34 \mathrm{~g} /$ ha, respectively. From that amount, $57.5 ; 52.8 ; 42.3 ; 68.5 ; 57.3 ; 37.6$; $70.7 ; 41.9 ; 73.6$ and $71.6 \%$, respectively, were deposited in the bulb.
\end{abstract}

Keywords: Allium cepa, nutrition of plant, macronutrients, micronutrients.

(Recebido para publicação em 10 de julho de 2015; aceito em 5 de janeiro de 2016)

(Received on July 10, 2015; accepted on January 5, 2016)

\begin{abstract}
$\mathrm{A}$ cebola (Allium cepa) é a terceira hortaliça em importância econômica para o Brasil (EPAGRI, 2013), tendo sido produzido 1,5 milhão de toneladas em 60 mil hectares em 2013 com rendimento médio de 25,9 t/ha (IBGE, 2014). A região Sul do Brasil concentra mais de $50 \%$ da produção e Santa Catarina
\end{abstract}

$30 \%$ da produção nacional. Dentre as cultivares de cebola, a Bola Precoce (Epagri 352 - Bola Precoce) é destaque, sendo cultivada em $60 \%$ da área em Santa Catarina e 50\% no Paraná e Rio Grande do Sul (Kurtz et al., 2009).

As curvas de absorção e acúmulo de nutrientes e de matéria seca possibilitam determinar as épocas de maior exigência dos nutrientes, prevenir deficiências, estimar a exportação e o retorno de nutrientes ao solo (May et al., 2008), proporcionando recomendação de adubação com eficiência agronômica e minimização dos riscos ambientais (Villas Boas et al., 2001; Furlani \& 
Purqueiro, 2010).

As exigências nutricionais podem variar entre cultivares de cebola e a extração de nutrientes e sua relação com o crescimento pode ser diferente em razão do tipo de solo, do sistema de cultivo, e ainda, variar segundo a produtividade e o ciclo da cultura (Vidigal et al., 2003).

A cebola tem crescimento lento até próximo a metade do ciclo (Haag et al., 1970; Wiedenfeld, 1994) e, a partir desta fase, quando tem início a bulbificação (Gamiely et al., 1991) e a translocação de fotoassimilados e outros compostos para o bulbo, ocorre rápido acúmulo de massa seca nessa parte da planta (Brewster, 2008). Vidigal et al. (2010), avaliando a cultivar Alfa Tropical em ciclo de verão com 130 dias em sistema de transplantio em Minas Gerais, observaram crescimento lento no inicio do ciclo com acúmulo de massa seca inferior a $10 \%$ até aos 74 dias após a semeadura, sendo posteriormente intensificado, assim como a absorção de nutrientes. Ao final do ciclo estes autores observaram para a população de 700.000 plantas/ha e produtividade de $24 \mathrm{t} / \mathrm{ha}$ uma absorção em kg/ha de 148,2 (N); 23,3 (P); 159,8 (K); 24,9 (S); 72,9 (Ca); $10,3(\mathrm{Mg}) ; 0,035(\mathrm{Cu}) ; 1,49(\mathrm{Fe}) ; 0,742$ $(\mathrm{Mn}) ;$ e $0,327(\mathrm{Zn})$. Os resultados sobre a quantidade de macronutrientes acumulada pela cebola são variáveis entre os autores (N: 63,0 a 148,2 kg/ha; P: 8,9 a $27,8 \mathrm{~kg} / \mathrm{ha} ; \mathrm{K}: 38,0$ a $159,8 \mathrm{~kg} / \mathrm{ha}$; Ca: 24,3 a $72,9 \mathrm{~kg} / \mathrm{ha} ; \mathrm{Mg}: 3,3$ a $14,8 \mathrm{~kg} /$ ha e $\mathrm{S}: 14,0$ a $37,5 \mathrm{~kg} / \mathrm{ha}$ ), dependendo da cultivar, tecnologia empregada e do local de cultivo (Porto et al., 2006, 2007; Santos, 2007; May et al., 2008; Vidigal et al., 2010).

No Sul do Brasil, para as cultivares de cebola mais difundidas, são escassas as pesquisas sobre a dinâmica de absorção de nutrientes, exigência nutricional e sua partição no sistema de transplante de mudas, que abrange mais de $70 \%$ da área cultivada. Assim, objetivou-se determinar o crescimento, acúmulo, partição e as taxas de absorção de nutrientes para a cultivar de cebola Bola Precoce no sistema de transplante na região Sul do Brasil.

\section{MATERIAL E MÉTODOS}

$\mathrm{O}$ experimento foi conduzido em campo na Estação Experimental da EPAGRI, na safra 2011/12, no município de Ituporanga, na região do Alto Vale do Itajaí-SC $\left(27^{\circ} 22^{\prime} \mathrm{S}, 49^{\circ} 35^{\prime} \mathrm{O}, 475\right.$ $\mathrm{m}$ de altitude). No período de condução do experimento as médias mensais de temperaturas foram de 12,$7 ; 13,8 ; 14,8$; 18,9 e $19,5^{\circ} \mathrm{C}$, de insolação (horas de brilho solar) foram 95, 114, 127, $168 \mathrm{e}$ 196 horas e as chuvas foram de 233,355 , 260, 133 e $85 \mathrm{~mm}$, respectivamente, para os meses de julho, agosto, setembro, outubro e novembro.

A área experimental está sendo cultivada desde 1995 em rotação de culturas, tendo a cebola como cultura principal no sistema de manejo do solo com cultivo mínimo. Antes do plantio da cebola, a área experimental foi cultivada com milheto (Pennisetum glaucum) como planta de cobertura. O solo do experimento é classificado como Cambissolo Húmico (Embrapa, 2006) de textura média (320 g/kg de argila). As amostras de solo coletadas de 0 a $20 \mathrm{~cm}$ na implantação do experimento apresentaram 5,6 de $\mathrm{pH}-\mathrm{H}_{2} \mathrm{O} ; 31 \mathrm{~g} / \mathrm{kg}$ de M.O.; $72 \mathrm{mg} / \mathrm{kg}$ de P; $0,66 \mathrm{cmol}_{\mathrm{c}} / \mathrm{kg}$ de $\mathrm{K} ; 6,0 \mathrm{cmol}_{\mathrm{c}} / \mathrm{kg} \mathrm{de} \mathrm{Ca} ; 3,1 \mathrm{cmol}_{\mathrm{c}} / \mathrm{kg}$ de $\mathrm{Mg} ; 14,7 \mathrm{cmol} / \mathrm{kg}$ de CTC; $0,0 \mathrm{cmol}^{\prime}$ $\mathrm{kg}$ de $\mathrm{Al} ; 66,6 \%$ de $\mathrm{V}$.

$\mathrm{O}$ delineamento experimento foi em blocos casualizados, com 18 tratamentos, correspondentes a cada época de avaliação/coleta da cultivar de cebola Bola Precoce (EPAGRI 352 - Bola Precoce) e cinco repetições (Fayad et al., 2002; Vidigal et al., 2010). No tempo zero foram avaliadas as mudas transplantadas. As demais coletas das plantas foram realizadas em intervalos de sete dias, aos $7,14,21,28,35,42$, $49,56,63,70,77,84,91,98,105,112$ e 119 dias após o transplantio (DAT). Os tratamentos (épocas de coleta) foram distribuídos aleatoriamente no bloco que correspondeu a uma linha útil de plantio no centro e duas linhas de bordadura, sendo uma de cada lado. $O$ espaçamento usado foi de $0,10 \mathrm{~m}$ entre plantas e 0,40 $\mathrm{m}$ entre as linhas, totalizando 250.000 plantas por hectare. Cada parcela foi constituída de 27 plantas $\left(1,1 \mathrm{~m}^{2}\right)$ e para cada avaliação foram coletadas as cinco plantas centrais de cada parcela.

As mudas foram previamente produzidas em canteiros, conforme sistema de produção vigente no estado de Santa Catarina (EPAGRI, 2013) e transplantadas aos 80 dias após a emergência, selecionando-se aquelas com diâmetro do pseudo-caule de 5-6 mm. Para o transplantio das mudas, os sulcos de plantio $(5 \mathrm{~cm}$ de largura e $10 \mathrm{~cm}$ de profundidade) foram abertos com o auxílio do equipamento "rotocaster" acoplado a um motocultivador. A semeadura foi realizada em 04/05/2011, o transplantio em 22/07/2011 e a colheita em 18/11/2011, quando $70 \%$ das plantas apresentaram tombamento da parte aérea (estalamento).

A adubação de base foi realizada imediatamente antes do transplantio, conforme recomendação de reposição da CQFS RS-SC (2004) com 90 kg/ha de $\mathrm{K}_{2} \mathrm{O}$ na forma de $\mathrm{KCl}$, distribuídos a lanço; $120 \mathrm{~kg} /$ ha de $\mathrm{P}_{2} \mathrm{O}_{5}$, na forma de super fosfato triplo e $20 \mathrm{~kg} / \mathrm{ha}$ de $\mathrm{N}$ na forma de nitrato de amônio. O superfosfato triplo e o nitrato de amônio foram incorporados no sulco de plantio. Em cobertura, foram aplicados $55 \mathrm{~kg} / \mathrm{ha}$ de $\mathrm{N}$ (nitrato de amônio) a lanço, aos 30,60 e 90 DAT, conforme indicado por Kurtz et al. (2012).

O controle de plantas espontâneas, de pragas e de doenças foi efetuado com agroquímicos, considerando dosagens e registros do Ministério da Agricultura para a cultura da cebola. No controle de plantas espontâneas, foram efetuadas duas aplicações de herbicidas (ioxynil e pendimetalina) e duas capinas manuais. Para o controle de pragas, especialmente do tripes (Thrips tabaci), foram realizadas três aplicações de inseticidas (lambdacyhalothrin, deltametrina e imidacloprid). Para o controle de doenças fúngicas, principalmente de míldio (Peronospora destructor) e alternaria (Alternaria solani), foram realizadas cinco pulverizações de fungicidas (metalaxyl + clorotalonil, iprodione e tebuconazole).

As plantas foram analisadas inteiras até o início da bulbificação e divididas em parte aérea e bulbos após esse período. O sistema radicular não foi avaliado. Após a coleta, as plantas foram lavadas com água deionizada e secas em estufa de circulação forçada de ar a $65^{\circ} \mathrm{C}$ até atingirem massa constante. Em seguida, foi realizada a pesagem para determi- 
nação do acúmulo de massa seca das plantas e trituradas em partículas com diâmetro inferior a $1 \mathrm{~mm}$ em moinho tipo Willey. Posteriormente foram determinados os teores de $\mathrm{N}, \mathrm{P}, \mathrm{K}, \mathrm{Ca}, \mathrm{Mg}$, $\mathrm{Zn}, \mathrm{Fe}, \mathrm{Cu}$ e B, conforme Tedesco et al. (1995). A partir dos valores da massa seca da parte vegetativa e do bulbo e os respectivos teores de nutrientes, foram calculados (teor x massa) o seu acúmulo nesses órgãos.

A taxa de crescimento absoluto foi obtida pela derivada primeira da equação ajustada à massa seca da planta (Benincasa, 2003). A partir dos valores da massa seca da parte vegetativa e do bulbo e os respectivos teores de N, P, $\mathrm{K}, \mathrm{Ca}, \mathrm{Mg}, \mathrm{Zn}, \mathrm{Fe}, \mathrm{Cu}, \mathrm{B}$ e $\mathrm{Mn}$ nestes órgãos, foram calculados os acúmulos de nutrientes nos diferentes órgãos. A taxa de absorção diária de cada nutriente foi obtida pela derivada primeira da equação ajustada ao conteúdo de cada nutriente na planta em função dos DAT das mudas (Fayad et al., 2002). O total acumulado pela planta correspondeu à soma das quantidades presentes na parte aérea e no bulbo, e representou a exigência nutricional da cebola em macro e micronutrientes. Assim, para o cálculo da quantidade exportada por área, considerou-se uma população de 250.000 plantas por hectare.

Todas as variáveis determinadas foram submetidas às análises de variância e, havendo significância $(\mathrm{p}<0,05)$, realizou-se análise de regressão, onde foram testados grupos de equações contendo dois, três e quatro parâmetros. Foi selecionado o modelo matemático com base no significado biológico e de maior coeficiente de determinação $\left(\mathrm{R}^{2}\right)$. A equação de modelo logístico sigmoidal não linear de três parâmetros $\mathrm{y}=\mathrm{a} /\{1+\exp [-(\mathrm{x}-\mathrm{x} 0) / \mathrm{b}]\}$ foi a que apresentou o melhor ajuste no geral e foi obtida com o emprego do programa gráfico SigmaPlot 11.0.

\section{RESULTADOS E DISCUSSÃO}

O acúmulo de massa seca (MS) das plantas de cebola foi lento até metade do ciclo (60 DAT), acumulando 3,9 g/ planta, o que representou $16 \%$ da MS total acumulada (Figura 1A). Após esse período, quando iniciou a fase de bulbificação, o acúmulo de MS foi intensificado (61 aos $119 \mathrm{DAT})$, acumulando $20,7 \mathrm{~g}$ /planta, equivalente a $84 \%$ da MS total acumulada. Acúmulos lentos de MS no início do ciclo também foram observados por outros autores, evidenciando que este comportamento ocorre independente da cultivar utilizada e do local de cultivo (Pôrto et al., 2007; May et al., 2008; Vidigal et al., 2010).
Da mesma forma, Vidigal et al. (2010) também observaram acúmulo rápido de MS a partir do início da bulbificação, aos 88 dias após a semeadura para a cultivar Alfa Tropical, o que também foi verificado para outras cultivares e condições de cultivo (Haag et al.,1970; Wiedenfeld, 1994; INIA, 2005). Ao final do ciclo, as plantas acumularam 24,6 g/ planta de MS total, sendo 9,29 g na parte aérea e 15,33 g no bulbo. A massa fresca média de bulbo foi de 149,4 g/planta e o rendimento total de bulbos foi de $37,34 \mathrm{t} / \mathrm{ha}$. Esse resultado foi superior aos rendimentos médios obtidos nessa safra que foram de 24,0 e 20,1 t/ha para o estado de Santa Catarina e do Brasil, respectivamente (IBGE, 2014).

Para uma população de 250.000 plantas/ha e rendimento de $37,34 \mathrm{t} / \mathrm{ha}$ de bulbos frescos, a produção foi 6.155 $\mathrm{kg} / \mathrm{ha}$ de MS total, distribuídos em $2.324 \mathrm{~kg} / \mathrm{ha}$ na parte aérea e $3.831 \mathrm{~kg} /$ ha nos bulbos, ou seja, 38 e $62 \%$, respectivamente. Resultados semelhantes foram obtidos por Pôrto et al. (2006), avaliando o híbrido Optima em sistema de semeadura direta obtendo as contribuições de 30 e $70 \%$, respectivamente, para a parte aérea e bulbos. Para Vidigal et al. (2002), em sistema de transplante de mudas, as contribuições de parte aérea e bulbo foram de aproximadamente 36 e $64 \%$, respectivamente, semelhantes

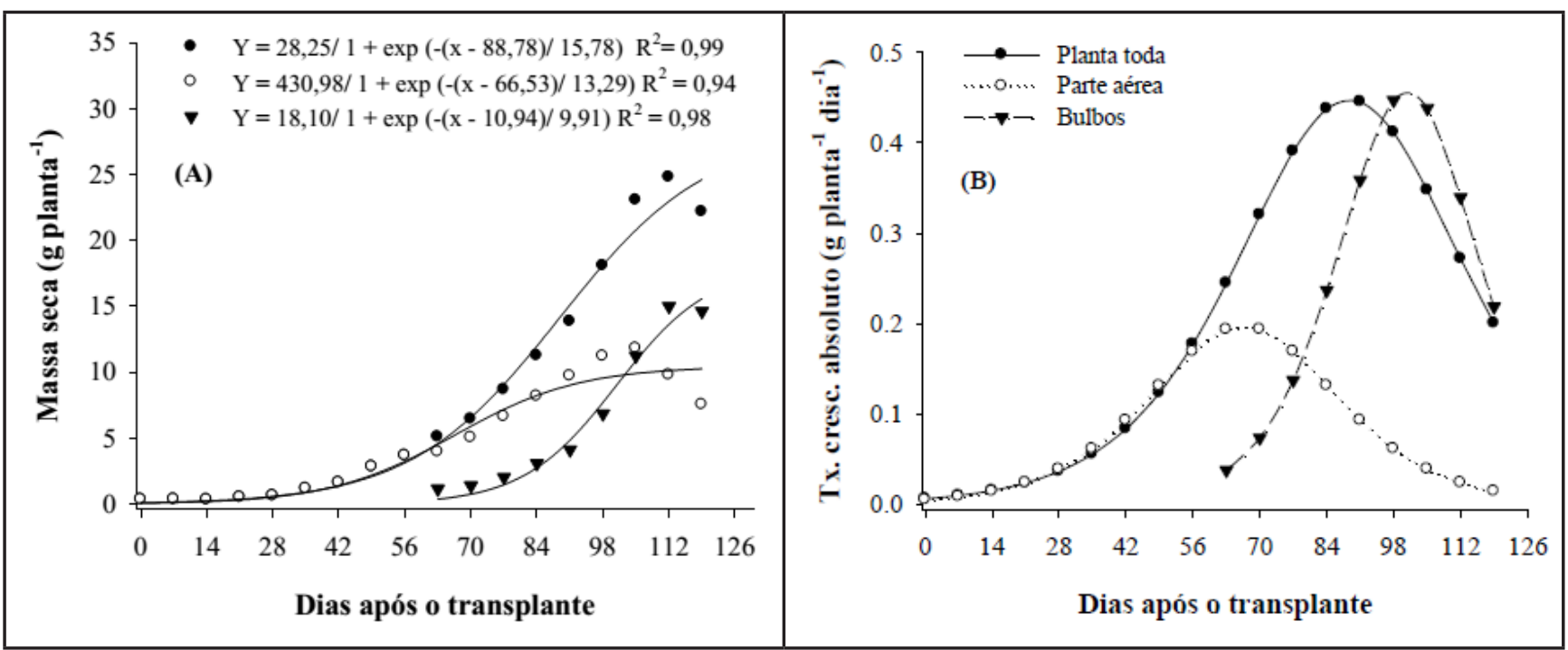

Figura 1. Acúmulo de massa seca $(\mathrm{A})$ e taxa de crescimento absoluto $(\mathrm{B})$ na planta toda $(\bullet)$, parte aérea (O) e bulbos ( $\boldsymbol{\nabla})$ de plantas de cebola da cv. Bola Precoce cultivada em sistema de transplante \{dry matter accumulation (A) and absolute growth rate (B) in the whole plant $(\bullet)$, aerial part $(\mathrm{O})$ and bulbs $(\boldsymbol{\nabla})$ of onion plants cv. Bola Precoce cultivated in transplanting system $\}$. Ituporanga, Epagri/UFPR, 2012. 


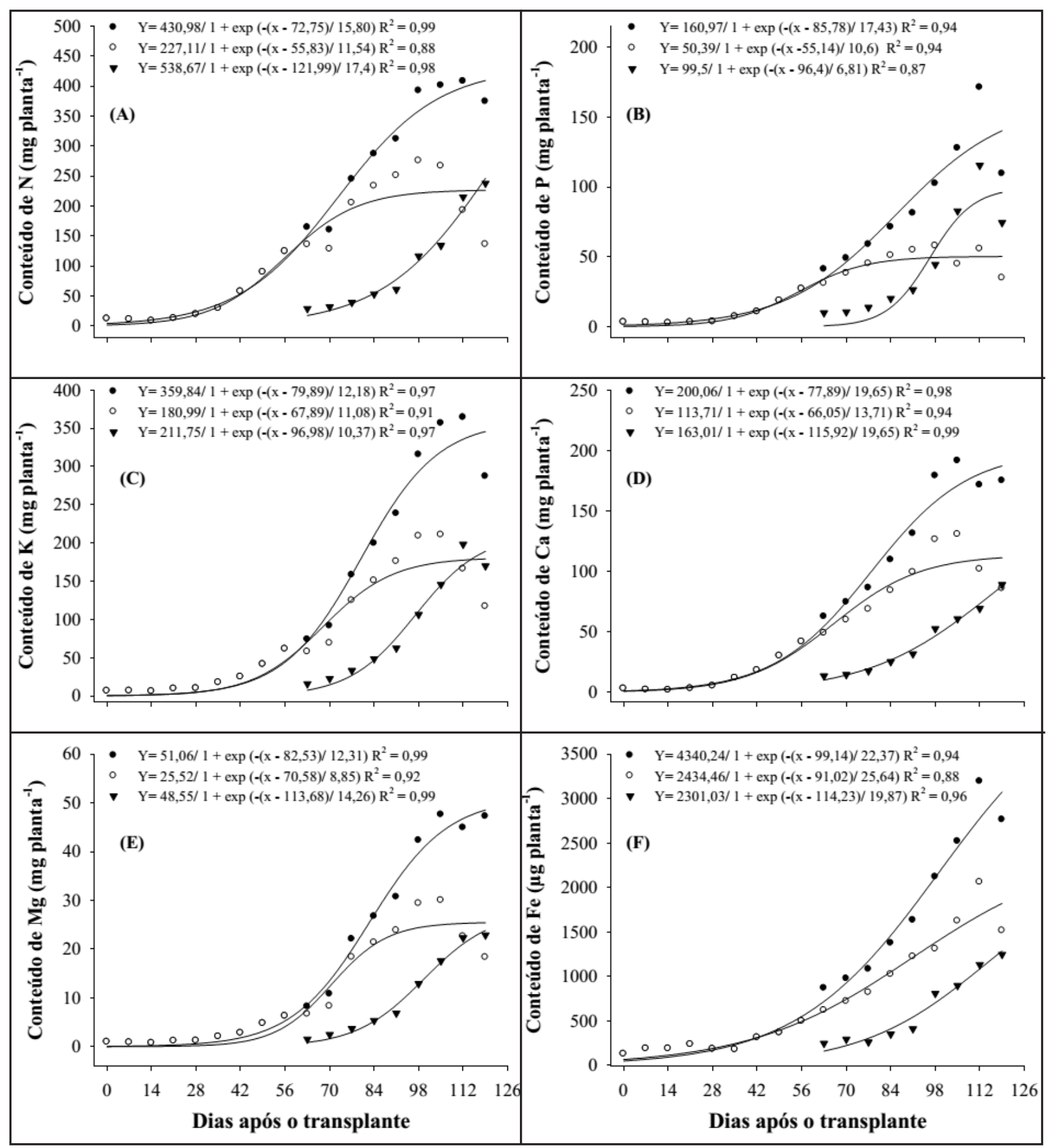

Figura 2. Conteúdo de N (A), P (B), K (C), Ca (D), Mg (E) e Fe (F) na planta toda (•), parte aérea (O) e bulbos ( $)$ ) de plantas de cebola da cv. Bola Precoce cultivada em sistema de transplante \{content of N (A), P (B), K (C), Ca (D), Mg (E) and Fe (F) in the whole plant (•), aerial part $(\mathrm{O})$ and bulbs $(\boldsymbol{\nabla})$ of onion plants cv. Bola Precoce cultivated in transplanting system $\}$. Ituporanga, Epagri/UFPR, 2012.

ao presente estudo.

A taxa máxima de crescimento absoluto para a planta toda ocorreu aos 89 DAT, quando atingiu $0,447 \mathrm{~g} / \mathrm{planta} /$ dia de MS (111,75 kg/ha/dia), desacelerando nos 30 dias que antecederam a colheita (Figura 1B). Para o bulbo, que iniciou sua formação aos 60 DAT, a taxa de crescimento máxima ocorreu aos 101 DAT com acúmulo de $0,46 \mathrm{~g} /$ planta/dia (115 kg/ha/dia de MS). A partir dos 96 DAT até a colheita, a taxa de crescimento absoluto da planta inteira foi menor que o alocado no bulbo diariamente, indicando redistribuição de fotoassimilados, principalmente das folhas para os bulbos (Figura 1B). Isso também foi observado por Pôrto et al. (2006, 2007) e Vidigal et al. (2010). Isso concorda com Brewster (2008), que afirma que na época de desenvolvimento 


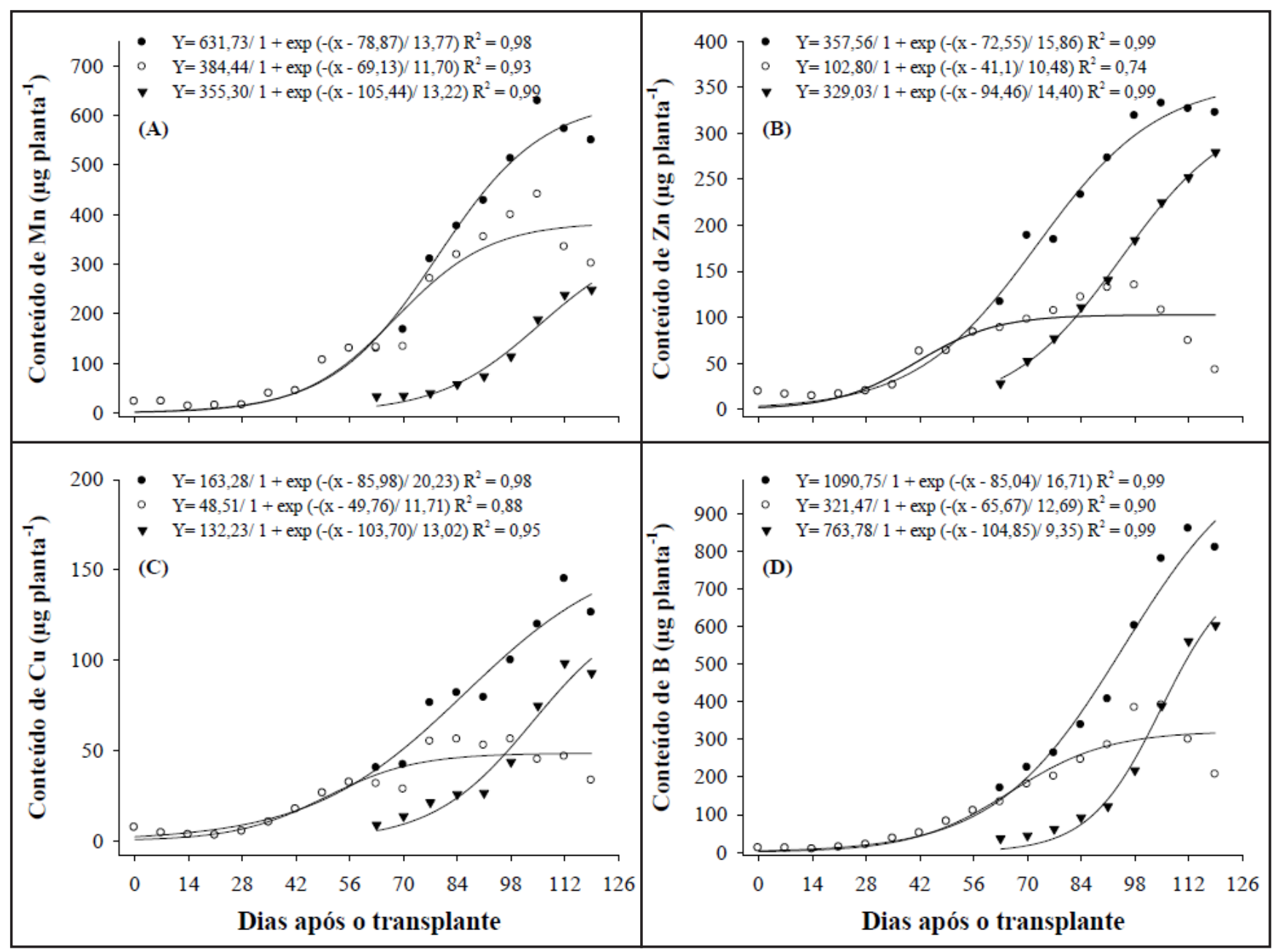

Figura 3. Conteúdo de Mn (A), Zn (B), Cu (C) e B (D) na planta toda (•), parte aérea (०) e bulbos ( $\mathbf{\nabla})$ de plantas de cebola da cv. Bola Precoce cultivada em sistema de transplante \{content of Mn (A), Zn (B), Cu (C) and B (D) in the whole plant (•), aerial part (०) and bulbs ( $\boldsymbol{\nabla})$ of onion plants cv. Bola Precoce cultivated in transplanting system $\}$. Ituporanga, Epagri/UFPR, 2012.

dos bulbos há translocação de fotoassimilados e outros compostos das folhas para os bulbos, resultando na redução da massa seca das folhas e aumento na massa seca dos bulbos.

As curvas de absorção de nutrientes para a planta de cebola tiveram comportamento similar às curvas de acúmulo de matéria seca, ajustando-se ao modelo sigmoidal (Figuras 2 e 3). No entanto, houve acúmulo proporcionalmente menor de MS na primeira metade do ciclo $(16 \%)$, do que a absorção da maioria dos nutrientes, exceto para $\mathrm{K}, \mathrm{Mg}$ e $\mathrm{B}$, cujos acúmulos nesta fase foram de 13,2, 11,2 e 10,8\%, respectivamente (Tabela 1).

De maneira geral, houve grandes acúmulos e maiores taxas de absorção de nutrientes (Figuras 4 e 5) na segunda metade do ciclo da cultura, durante a fase de bulbificação (61-119 DAT). Es- ses resultados são similares aos obtidos por Pôrto et al. (2006) com o híbrido Optima em semeadura direta no estado de São Paulo.

As taxas diárias de acúmulo de nutrientes, exceto de $\mathrm{Fe}$, na parte aérea, aumentaram até início da bulbificação e decresceram após esta fase (Figuras 4 e 5). Isso mostra que a planta de cebola prioriza a alocação de fotoassimilados e nutrientes para o bulbo, assim que inicia a formação desse órgão (Brewster, 2008).

$\mathrm{O} \mathrm{N}$ foi o elemento mais absorvido pelas plantas de cebola, estimado em $409 \mathrm{mg} /$ planta, o que equivale a uma extração de 101,4 kg/ha de N (Figura 2A; Tabela 1). A quantidade absorvida de $\mathrm{N}$ foi $35,2 \%$ superior a quantidade adicionada na adubação mineral (75 $\mathrm{kg} / \mathrm{ha}$ ), demonstrando uma contribuição importante da matéria orgânica do solo no fornecimento de N. May et al. (2008), estudando o híbrido Optima em sistema de semeadura direta, também obtiveram o $\mathrm{N}$ como nutriente mais absorvido (106,36 mg/planta). Isso também ocorreu com Santos (2007) para cultivar Alfa São Francisco em sistema de transplante na região Nordeste, observando acúmulo de $103,4 \mathrm{~kg} / \mathrm{ha}$ de N. No entanto, outros autores obtiveram que o $\mathrm{N}$ foi o segundo nutriente mais absorvido, geralmente superado pelo K (Haag et al., 1970; Pôrto et al., 2006, 2007; Vidigal et al., 2010). Essas diferenças quanto ao nutriente mais absorvido pela cebola, se o $\mathrm{N}$ ou o $\mathrm{K}$, são devidas à variação em função da cultivar, solo, clima ou sistema de cultivo.

Do total de $\mathrm{N}$ acumulado pela planta, $43 \%$ foram alocados para a parte 
aérea e 57\% para os bulbos (Tabela 1), confirmando os resultados obtidos por Pôrto et al. (2006). Antes de iniciar a bulbificação, as plantas acumularam menos de $1 / 3(27 \%)$ do $\mathrm{N}$ total, enquanto a maior demanda (73\%) ocorreu após este período e a taxa máxima de absorção foi aos 73 DAT para a planta toda com 6,82 $\mathrm{mg} / \mathrm{planta} /$ dia (Figura 4A). No bulbo, o $\mathrm{N}$ também foi o elemento mais acumulado e teve maiores taxas de acúmulo e demanda crescente até no final do ciclo
(119 DAT). A quantidade de $\mathrm{N}$ maior no bulbo do que na parte aérea no final do ciclo pode ser atribuído à redistribuição do $\mathrm{N}$ presente na parte aérea.

$\mathrm{O} \mathrm{P}$ foi o quarto elemento mais absorvido pela planta de cebola depois do

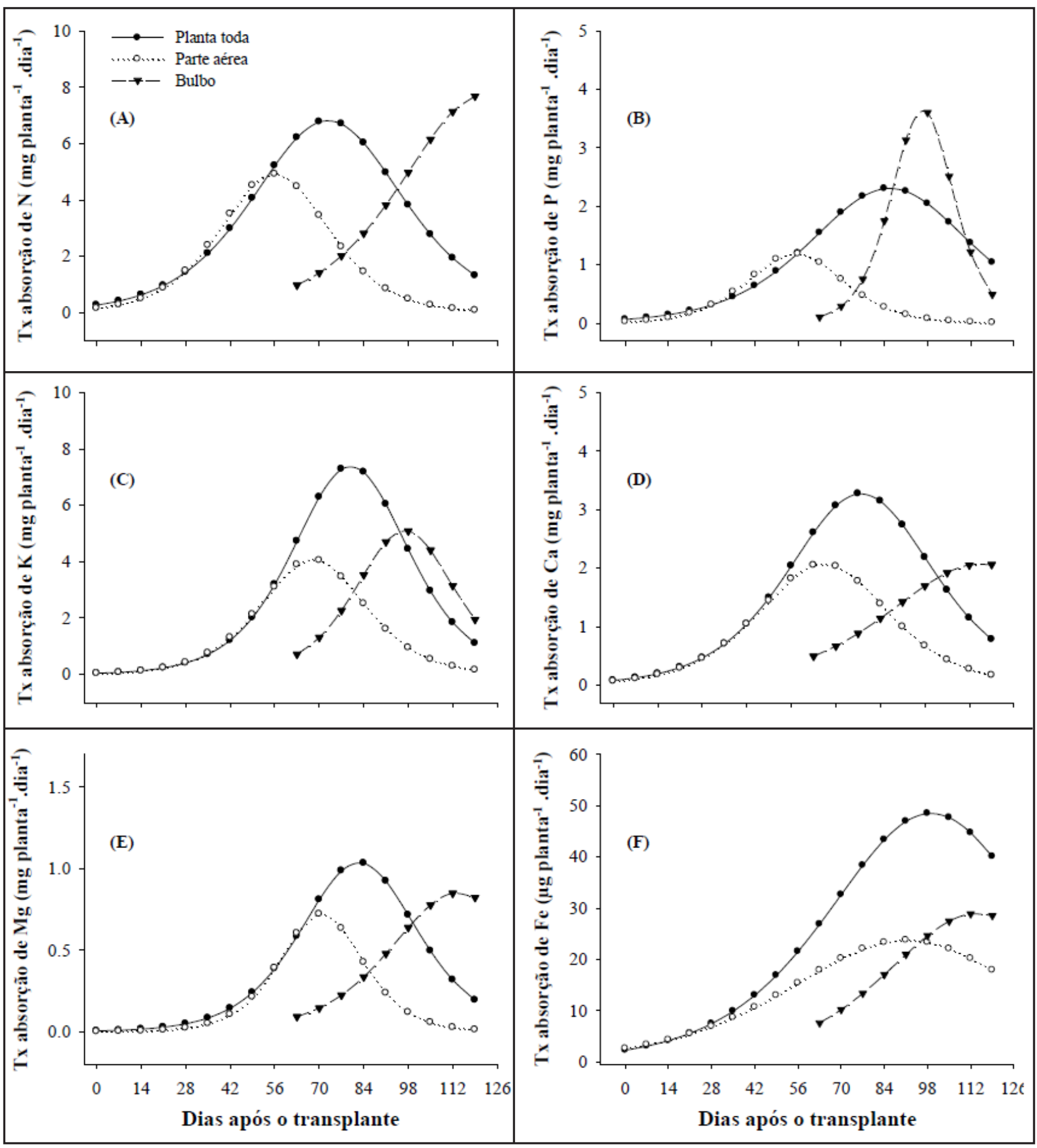

Figura 4. Taxas diárias de absorção de $\mathrm{N}(\mathrm{A}), \mathrm{P}(\mathrm{B}), \mathrm{K}(\mathrm{C}), \mathrm{Ca}(\mathrm{D}), \mathrm{Mg}(\mathrm{E})$ e Fe (F) na planta toda $(\bullet)$, parte aérea (o) e bulbos ( $\mathbf{\nabla})$ de plantas de cebola da cv. Bola Precoce, cultivada em sistema de transplante \{daily absorption rates of N (A), P (B), K (C), Ca (D), Mg $(\mathrm{E})$ and $\mathrm{Fe}(\mathrm{F})$ in the whole plant $(\bullet)$, aerial part $(\mathrm{O})$ and bulbs $(\boldsymbol{\nabla})$ of onion plants cv. Bola Precoce cultivated in transplanting system $\}$. Ituporanga, Epagri/UFPR, 2012. 


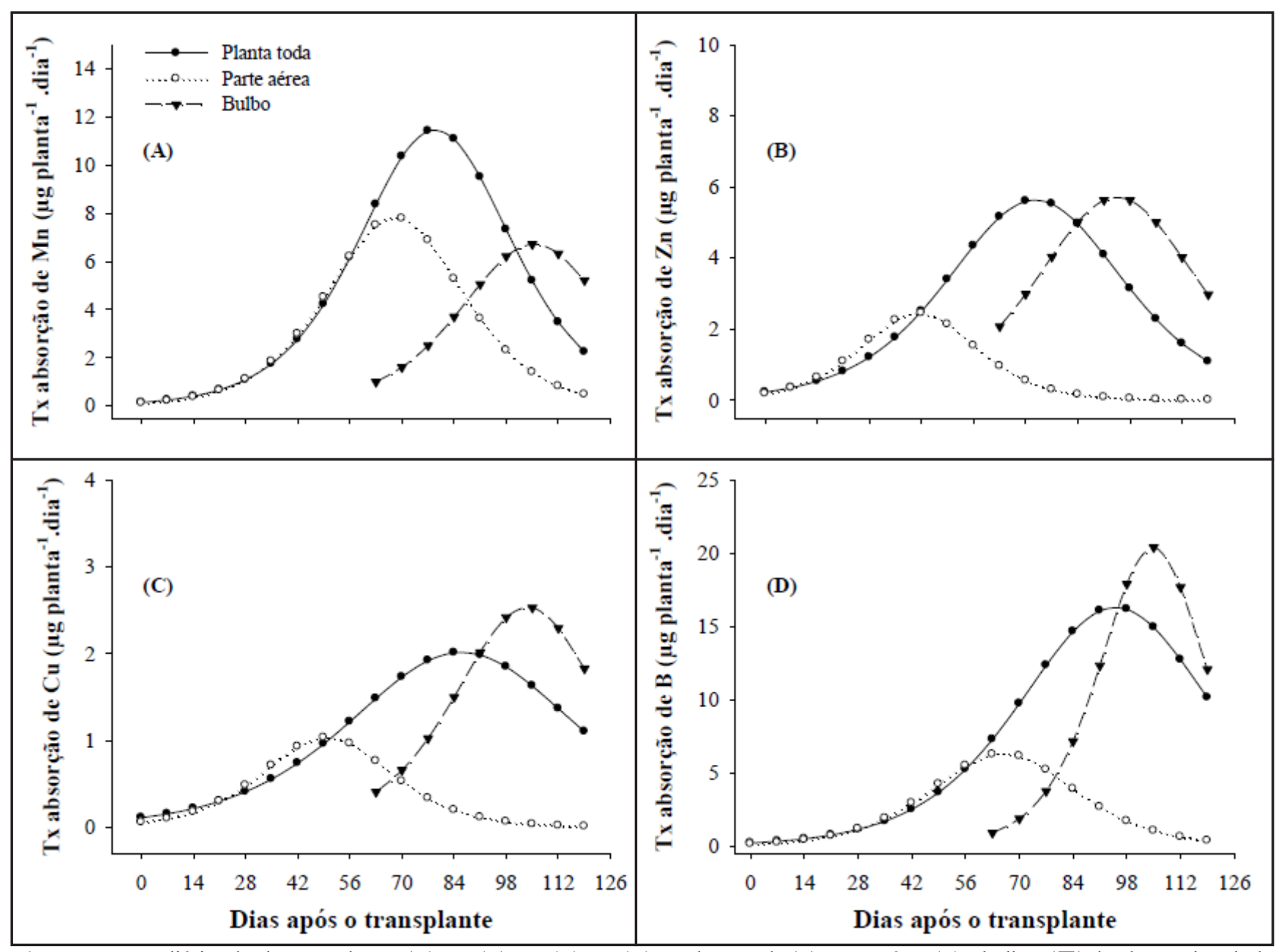

Figura 5. Taxas diárias de absorção de $\mathrm{Mn}(\mathrm{A}), \mathrm{Zn}(\mathrm{B}), \mathrm{Cu}(\mathrm{C})$ e B (D) na planta toda $(\bullet)$, parte aérea (o) e bulbos $(\boldsymbol{\nabla})$ de plantas de cebola da cv. Bola Precoce cultivada em sistema de transplante \{daily absorption rates of Mn (A), Zn (B), Cu (C) and B (D) in the whole plant $(\bullet)$, aerial part (०) and bulbs $(\boldsymbol{\nabla})$ of onion plants cv. Bola Precoce cultivated in transplanting system $\}$. Ituporanga, Epagri/UFPR, 2012.

N, K e Ca com acúmulo de 140,13 mg/ planta, equivalente a $34,9 \mathrm{~kg} / \mathrm{ha}$ (Figura 2B). Dentre os macronutrientes, o P foi o que, proporcionalmente, apresentou maior acúmulo no bulbo com $69 \%$, enquanto que a parte aérea contribuiu com $31 \%$. May et al. (2008) também observaram maior acúmulo de $\mathrm{P}$ no bulbo na ordem de 56 e $61 \%$, respectivamente, para os híbridos Superex e Optima.

As taxas máximas de absorção, considerando a planta toda, ocorreram aos 86 DAT e verificou-se uma taxa de alocação de P para o bulbo elevada no período próximo aos $96 \mathrm{DAT}$, momento que ocorreu a taxa máxima de alocação do elemento (Figura 4B). Do total de $\mathrm{P}$ absorvido pela planta, $83 \%$ foi no período de bulbificação (Tabela 1). Após o início da bulbificação, decresceu rapidamente a taxa de alocação de P para a parte aérea, o que também foi observado por Pôrto et al. (2007). Segundo Araújo \& Machado (2006), ocorre intenso processo de redistribuição de $\mathrm{P}$ dos tecidos vegetativos para órgãos reprodutivos. Para esses autores, nas culturas de grãos, a proporção entre a quantidade de nutrientes nos grãos e a quantidade de nutrientes na biomassa também foi superior para o $\mathrm{P}$ que os demais macronutrientes, indicando uma redistribuição preferencial de P para os órgãos drenos.

$\mathrm{O} \mathrm{K}$ foi o segundo nutriente mais acumulado pela cebola, totalizando 346 $\mathrm{mg} /$ planta $(86,5 \mathrm{~kg} / \mathrm{ha})$, sendo superado apenas pelo N (Figura 2C), semelhantes aos resultados de May et al. (2008) e Santos (2007). Outros autores, no entanto, encontraram que o $\mathrm{K}$ foi o elemento mais absorvido pela cebola (Haag et al.,
1970; Pôrto et al., 2006, 2007; Vidigal et al., 2010), o que reflete o efeito da cultivar, técnicas de manejo e ambiente de cultivo na absorção do $\mathrm{K}$, conforme já discutido para o $\mathrm{N}$.

Do total acumulado de $\mathrm{K}$ ao final do ciclo da cultura, o bulbo contribuiu com 53\%, (182,7 mg/planta (Tabela 1). A absorção de $\mathrm{K}$ foi concentrada no período de bulbificação com $87 \%$ do total acumulado nessa fase. A taxa máxima de absorção pela planta toda foi aos 80 DAT com 7,38 mg/planta/dia, o equivalente a $1,84 \mathrm{~kg} / \mathrm{dia}$ de K. Para o bulbo, a taxa máxima de alocação ocorreu 17 dias mais tarde aos 97 DAT (Figura 4C). O potássio desempenha um importante papel na abertura dos estômatos e regulação do potencial osmótico das células vegetais (Taiz \& Zeiger, 2009). Esse último processo provavelmente explica 
a grande demanda de K na bulbificação, quando promove a redução do potencial osmótico favorecendo a entrada de água e de fotoassimilados, contribuindo para o enchimento dos bulbos.

Considerando a prática da adubação, é necessária uma disponibilidade de aproximadamente $1,84 \mathrm{~kg} /$ dia de $\mathrm{K}$ (ou 2,21 de $\mathrm{K}_{2} \mathrm{O}$ ) no pico de absorção deste nutriente (80 DAT). Esse valor é semelhante ao do $\mathrm{N}$, que demandou $1,71 \mathrm{~kg} /$ ha por dia no período de maior demanda (73 DAT). Esses dados são importantes orientadores para definição da época de aplicação desses nutrientes para a cultura da cebola, uma vez que apresentam mobilidade, principalmente em solos arenosos ou de baixa CTC (Sangoi et al., 2003; Cantarella, 2007; Ernani et al., 2007) e se forem aplicados muito antes desta data podem não atender a demanda da planta. Considerando o início da bulbificação aos 60 DAT, ou seja, 13 e 20 dias antes da maior taxa de absorção de N (73 DAT) e K (80 DAT), respectivamente, essa seria a fase da cultura sugerida para aplicação das adubações de cobertura com estes nutrientes. Essa sugestão é diferente da CQFS RS/SC (2004) que recomendam todo $\mathrm{K}$ no plantio e a aplicação de cobertura com
N em uma única aplicação aos 45 DAT.

$\mathrm{O} \mathrm{Ca}$ foi o terceiro nutriente mais absorvido pela planta de cebola, com quantidade acumulada de $186,5 \mathrm{mg}$ / planta $(46,6 \mathrm{~kg} / \mathrm{ha})$ ao final do ciclo (Figura 2 D). A absorção de Ca foi diferente dos demais macronutrientes, pois foi acumulado mais da metade na parte aérea, $58 \%$ do total e no bulbo acumulou 42\% (Tabela 1). Isso concorda com Vidigal et al. (2010), que observaram acúmulo de 55 e $45 \%$, respectivamente, para parte aérea e bulbos. A distribuição do Ca é, portanto, resultado da redistribuição do nutriente ser praticamente nula (Hawkesford et al., 2012).

Outro fator que pode ter contribuído para essa diferença nas quantidades de Ca na parte aérea e bulbo é a competição com o K (Andriolo et al., 2010). O maior fluxo de K para o bulbo pode restringir a presença de Ca neste órgão. Com relação à dinâmica de absorção, observou-se que a taxa máxima de acúmulo foi aos 78 DAT para a planta toda e aos 116 DAT para o bulbo (Figura 4D). Assim, verifica-se uma maior demanda de $\mathrm{Ca}$ para o bulbo no fim do ciclo, demonstrado pela absorção de $79,4 \%$ do Ca total no período de bulbificação.

$\mathrm{O} \mathrm{Mg}$ foi o macronutriente menos absorvido pela cebola com 46,6 mg/ planta, totalizando $12,1 \mathrm{~kg} /$ ha (Figura 2E), sendo distribuídos em aproximadamente $43 \%$ na parte aérea e $57 \%$ no bulbo (Tabela 1). Vidigal et al. (2002) relataram valores semelhantes para a cultivar Alfa Tropical, que acumulou 47 e $53 \%$, respectivamente, para parte aérea e bulbo. Entretanto, May et al. (2008) observaram valores diferentes, em que a planta acumulou 56 e $44 \%$ para o híbrido Optima e 67,2 e 38,2\% para o híbrido Superex, respectivamente, para parte aérea e bulbo.

A maior taxa de absorção de $\mathrm{Mg}$ ocorreu aos 83 DAT na planta toda, e aos 112 DAT, a maior demanda para o bulbo, a exemplo do que ocorreu para $\mathrm{Ca}$ (Figura 4E). O Mg foi o macronutriente que apresentou a maior proporção de acúmulo no período de bulbificação, cerca de $89 \%$ do total. Esta grande demanda de Mg na bulbificação ocorre devido ao acúmulo de açucares no vacúolo de órgãos de reserva ser dependente do $\mathrm{Mg}$ e estimulado pelo K (Engels et al., 2012).

A absorção de micronutrientes ocorreu na seguinte sequência: $\mathrm{Fe}>\mathrm{B}>\mathrm{Mn}>\mathrm{Zn}>\mathrm{Cu}$ (Figura 2F; Figura 3; Tabela 1), com acúmulo ao final do ciclo de 761, 221, 150, 84 e $34 \mathrm{~g} / \mathrm{ha}$,

Tabela 1. Acúmulo de nutrientes na planta toda, no bulbo, por tonelada de bulbo, antes e após iniciar a bulbificação e período das taxas máximas de acúmulo de nutrientes pela cultivar de cebola Bola Precoce para uma produtividade de $37.343 \mathrm{~kg} / \mathrm{ha}$ (nutrient accumulation in the whole plant, bulb, per ton of bulb, before and after bulbification and the maximum rates of accumulation of nutrients by onion Bola Precoce considering a productivity of 37,343 kg/ha). Ituporanga, Epagri/UFPR, 2012.

\begin{tabular}{|c|c|c|c|c|c|c|c|c|}
\hline \multirow{3}{*}{$\begin{array}{l}\text { Nutriente } \\
\text { Macronutrientes }\end{array}$} & \multicolumn{6}{|c|}{ Acúmulo nutrientes } & \multicolumn{2}{|c|}{ Tx. máxima acúmulo } \\
\hline & \multirow{2}{*}{$\begin{array}{c}\text { Planta toda } \\
(\mathrm{kg} / \mathrm{ha})\end{array}$} & \multicolumn{2}{|c|}{ Bulbo } & \multirow{2}{*}{$\begin{array}{c}\text { Tonelada } \\
\text { bulbo } \\
(\mathrm{kg} / \mathrm{t})\end{array}$} & \multirow{2}{*}{$\begin{array}{c}\text { Antes da } \\
\text { bulbificação }{ }^{2} \\
(\%)\end{array}$} & \multirow{2}{*}{$\begin{array}{c}\text { Após a } \\
\text { bulbificação } \\
(\%) \\
\text { (\%) }\end{array}$} & Planta & Bulbo \\
\hline & & $(\mathrm{kg} / \mathrm{ha})$ & $(\%)$ & & & & \multicolumn{2}{|c|}{ DAT $^{1}$} \\
\hline $\mathrm{N}$ & 101,4 & 58,3 & 57,5 & 2,72 & 27,0 & 73,0 & 73 & 119 \\
\hline $\mathrm{P}$ & 34,9 & 23,9 & 68,5 & 0,93 & 17,3 & 82,7 & 86 & 96 \\
\hline K & 86,5 & 45,7 & 52,8 & 2,32 & 13,2 & 86,8 & 80 & 97 \\
\hline $\mathrm{Ca}$ & 46,6 & 19,7 & 42,3 & 1,25 & 20,6 & 79,4 & 78 & 116 \\
\hline $\mathrm{Mg}$ & 12,1 & 7,0 & 57,3 & 0,33 & 11,2 & 88,8 & 83 & 112 \\
\hline Micronutrientes & (g/ha) & (g/ha) & & $(g / t)$ & & & & \\
\hline $\mathrm{Fe}$ & 761,2 & 285,9 & 37,6 & 20,38 & 16,8 & 83,2 & 99 & 114 \\
\hline $\mathrm{Mn}$ & 149,6 & 62,7 & 41,9 & 4,01 & 17,0 & 83,0 & 79 & 105 \\
\hline $\mathrm{Zn}$ & 84,1 & 61,9 & 73,6 & 2,25 & 27,3 & 72,7 & 73 & 94 \\
\hline $\mathrm{Cu}$ & 33,7 & 24,1 & 71,6 & 0,90 & 21,2 & 78,8 & 86 & 104 \\
\hline $\mathrm{B}$ & 220,6 & 155,9 & 70,7 & 5,91 & 10,8 & 89,2 & 95 & 105 \\
\hline
\end{tabular}

${ }^{1}$ Dias após o transplante (days after transplantating): ${ }^{2}$ Período do plantio aos 60 DAT (period from planting until 60 DAT); ${ }^{3}$ Período dos 61 DAT até a colheita, 119 DAT (period from 61 DAT until harvest, 119 DAT). 
respectivamente. Vidigal et al. (2010) observaram outra ordem na quantidade absorvida de micronutrientes: $\mathrm{Fe}>\mathrm{Mn}>\mathrm{Cu}>\mathrm{Zn}$, com a absorção de 630, 190, 30 e $21 \mathrm{~g} / \mathrm{ha}$, respectivamente, porém para a cultivar Alfa Tropical. Para os elementos Fe e Mn, observou-se maior acúmulo na parte aérea, 62 e $58 \%$, respectivamente, ao passo que $\mathrm{Zn}$, $\mathrm{Cu}$ e $\mathrm{B}$, foram acumulados em maior proporção nos bulbos com 74, 72 e 71\%, respectivamente. As maiores demandas dos micronutrientes também ocorreram na segunda metade do ciclo, no período de bulbificação com a absorção de 83, $83,73,79$ e $89 \%$ do total de Fe, Mn, $\mathrm{Zn}, \mathrm{Cu}$ e B, respectivamente. As taxas máximas de absorção situaram-se aos 99, 79, 73, 86 e 95 DAT para parte aérea e aos 114, 105, 94, 104 e 105 DAT para a alocação no bulbo, respectivamente, para $\mathrm{Fe}, \mathrm{Mn}, \mathrm{Zn}, \mathrm{Cu}$ e B (Figuras 4F, $5 \mathrm{~A}, 5 \mathrm{~B}, 5 \mathrm{C}$ e $5 \mathrm{D})$.

A taxa de alocação de micronutrientes no bulbo no final do ciclo foi superior à taxa média da planta para todos os nutrientes avaliados exceto Fe. Isso indica que, além da redistribuição dos nutrientes da parte aérea para os bulbos, os nutrientes absorvidos nessa fase de desenvolvimento foram depositados preferencialmente nos bulbos. Por se tratar de planta de bulbo e esse constituir parte da folha, provavelmente parte destes nutrientes foram absorvidos e acumulados diretamente no bulbo. Vidigal et al. (2010) observaram resultados semelhantes, onde a taxa de alocação de diversos nutrientes no bulbo foi superior a taxa observada para a planta toda.

A sequência de acúmulo de nutrientes verificada para a cultivar de cebola Bola Precoce em ordem decrescente foi $\mathrm{N}>\mathrm{K}>\mathrm{Ca}>\mathrm{P}>\mathrm{Mg}>\mathrm{Fe}>\mathrm{B}>\mathrm{Mn}>\mathrm{Zn}>\mathrm{Cu}$. Esses resultados foram semelhantes aos obtidos por Santos (2007) para a cultivar Alfa São Francisco. Por outro lado, outros autores tiveram resultados divergentes: May et al. (2008) constataram para o híbrido Optima a ordem $\mathrm{N}>\mathrm{Ca}>\mathrm{K}>\mathrm{Mg}>\mathrm{P}$ e para o híbrido Superex: $\mathrm{Ca}>\mathrm{N}>\mathrm{K}>\mathrm{Mg}>\mathrm{P}$. Vidigal et al. (2010) verificaram para a cultivar Alfa Tropical a seguinte ordem de extração $\mathrm{K}>\mathrm{N}>\mathrm{Ca}>\mathrm{S}>\mathrm{P}>\mathrm{Mg}>\mathrm{Fe}>\mathrm{Mg}>\mathrm{Cu}>\mathrm{Zn}$, que por sua vez foram semelhantes aos dados obtidos por Pôrto et al. (2006) para macronutrientes em trabalho com o hibrido Superex. Esses resultados evidenciam que as quantidades absorvidas de nutrientes e a sequência de acúmulo podem variar dependendo das cultivares e condições de cultivo.

Ao final do ciclo as plantas de cebola extraíram na planta inteira para cada tonelada de bulbos produzida 2,72; 0,$93 ; 2,32 ; 1,25 ; 0,33 \mathrm{~kg}$ e 20,$38 ; 4,01$; 2,25; 0,90 e $5,91 \mathrm{~g}$, respectivamente para N, P, K, Ca, Mg, Fe, Mn, Zn, Cu e B (Tabela 1). Dessa quantidade, 57,5; 68,$5 ; 52,8 ; 42,3 ; 57,3 ; 37,6 ; 41,9 ; 73,6$; 71,6 e $70,7 \%$, respectivamente, foram acumulados no bulbo. É importante salientar que, ao retirar a planta inteira da lavoura por ocasião da colheita, prática bastante comum no Sul do Brasil, deve-se considerar como exportação o total de nutrientes acumulado pelas plantas. Caso contrário, quando é realizada a "toalete" na lavoura e retirados somente os bulbos na colheita, considera-se como exportação, os nutrientes que fazem parte dos mesmos.

Os resultados obtidos permitem concluir que a cultivar de cebola Bola Precoce no sistema de transplante apresentou crescimento lento e demanda baixa por nutrientes na primeira metade do ciclo. Durante a bulbificação (61-119 DAT) o crescimento e a absorção de nutrientes foram intensos com acúmulo de $84 \%$ da MS total e entre 73 a $89 \%$ do total de nutrientes. A sequência de acúmulo de nutrientes verificada em ordem decrescente foi $\mathrm{N}>\mathrm{K}>\mathrm{Ca}>\mathrm{P}>\mathrm{Mg}>\mathrm{Fe}>\mathrm{B}>\mathrm{Mn}>\mathrm{Zn}>\mathrm{Cu}$, com 101,$4 ; 86,5 ; 46,6 ; 34,5 ; 12,1 \mathrm{~kg} / \mathrm{ha}$ e $761 ; 221 ; 150 ; 84$; e $34 \mathrm{~g} /$ ha, respectivamente. Desta quantidade, 57,$5 ; 52,8$; 42,$3 ; 68,5 ; 57,3 ; 37,6 ; 70,7 ; 41,9 ; 73,6$ e $71,6 \%$, respectivamente, foram depositados no bulbo. As taxas máximas de absorção de nutrientes se concentraram na fase de bulbificação, aos $73,86,80$, 78, 83, 99, 79, 73, 86 e 95 DAT, para $\mathrm{N}, \mathrm{P}, \mathrm{K}, \mathrm{Ca}, \mathrm{Mg}, \mathrm{Fe}, \mathrm{Mn}, \mathrm{Zn}, \mathrm{Cu}$ e B, respectivamente.

\section{AGRADECIMENTOS}

Aos colaboradores técnico agrícola Marcelo Pitz e assistente de campo Olindo André, da Epagri, Estação Ex- perimental de Ituporanga-SC.

\section{REFERÊNCIAS}

ANDRIOLO, JL; JÄNISCHI, DI; SCHMITTI, OJ; PICIOI, MD; CARDOSO, FL; ERPENI, L. 2010. Doses de potássio e cálcio no crescimento da planta, na produção e na qualidade de frutas do morangueiro em cultivo sem solo. Ciência Rural 40: 267-272.

ARAÚJO, AP; MACHADO, CTT. 2006. Fósforo. In: FERNANDES, MS (ed). Nutrição mineral de plantas. Viçosa: SBCS, p.253-280.

BENINCASA, MAP. 2003. Análise de crescimento de planas. 2 ed. Jabotical, SP: Funep. 41p.

BREWSTER, JL. 2008. Onions and other vegetable alliums. 2 ed. Wellesbourne, UK. $455 \mathrm{p}$.

CANTARELLA, H. 2007. Nitrogênio. In: NOVAIS, RF; ALVAREZ, VH; BARROS, RLFE; CANTARUTTI, RB; NEVES, JCL. Fertilidade do Solo. Viçosa, MG: SBCS, p.375-470.

COMISÃO DE QUÍMICA E FERTILIDADE DO SOLO - CQFS RS/SC. 2004. Manual de adubação e calagem para os Estados do Rio Grande do Sul e de Santa Catarina. 10 ed. Porto Alegre, Sociedade Brasileira de Ciência do Solo/Núcleo Regional Sul/ Universidade Federal do Rio Grande do Sul. 394p.

EMBRAPA. 2006. Sistema brasileiro de classificação de solos. 2. ed. Rio de Janeiro: Embrapa Solos. 306p.

EMPRESA DE PESQUISA AGROPECUÁRIA E EXTENSÃO RURAL DE SANTA CATARINA - EPAGRI. 2013. Sistemas de produção para cebola: Santa Catarina (4 revisão). Florianópolis: Epagri. 106p. (Sistemas de Produção, 46).

ENGELS, C; KIRKBY, E; WHITE, P. 2012. Mineral nutrition, yield and source-sink relationships. In: MARSCHNER, H (ed). Mineral nutrition of higher plants. 3 ed. San Diego: Academic Press. p.85-133

ERNANI, PR; ALMEIDA, JA; SANTOS, FC. 2007. Potássio. In: NOVAIS, RF; ALVAREZ, VH; BARROS, RLFE; CANTARUTTI, RB; NEVES, JCL. Fertilidade do Solo. Viçosa: SBCS, p.551-594.

FAYAD, JA; FONTES, PCR; CARDOSO, AA; FINGER, FL; FERREIRA, FA. 2002. Absorção de nutrientes pelo tomateiro cultivado sob condições de campo e de ambiente protegido. Horticultura Brasileira 20: 90-94.

FURLANI, PR: PURQUEIRO, LFV. 2010. Avanços e desafios na nutrição de hortaliças. In: Nutrição de plantas: diagnose foliar em hortaliças. MELLO PRADO, R. Jaboticabal: UNESP. p.45-62.

GAMIELY, S; RANDLE, WM; MILLS, HA; SMITTLE, DA. 1991. Onion plant growth, bulb quality, and water uptake following ammonium and nitrate nutrition. HortScience 26: 1061-1063.

HAWKESFORD, M; HORST, W; KICHEY, T; LAMBERS, H; SCHJOERRING, J; 
MOLLER, IS; WHITE, P. 2012. Functions of macronutrients. In MARSCHNER, H. Mineral nutrition of higher plants. 3 ed. San Diego: Academic Press. p.171-178.

HAAG, HP; HOMA, P; KIMOTO, T. 1970. Nutrição mineral de hortaliças. VIII. Absorção de nutrientes pela cultura da cebola. Anais... Escola superior de Agricultura "luiz de Queiroz”, Piracicaba. p. 143-153.

IBGE - Instituto Brasileiro de Geografia e Estatística. 2014. Sistema IBGE de recuperação automática-SIDRA. Disponível em http:www.sidra.ibge.gov.br. Acessado em 15 de dezembro, 2014.

INIA - Instituto Nacional de Investigacion Agropecuária do Uruguai. 2005. Tecnología para la producción de cebolla. Montevideo: Unidad de agronegocios y difusión del INIA. 247p. (Boletin de Divulgacion, 88).

KURTZ, C; ERNANI, PR; COIMBRA, JLM; PETRY, E. 2012. Rendimento e conservação de cebola alterados pela dose e parcelamento de nitrogênio em cobertura. Revista Brasileira de Ciência do Solo 36: 865-876.

KURTZ, C; SCHMITT, DR; MENEZES JÚNIOR, FOG; WERNER, H; GONÇALVES, PAS; LANNES, SD; MISSIO, VC. 2009. Relatório de avaliação dos impactos das tecnologias e ações geradas pela Epagri. Ituporanga: Epagri, 11p. (Relatório Balanço Social, 2009).

MAY, A; CECÍlIO FILHO, AB; PORTO, DRQ; VARGAS, PF; BARBOSA, JC. 2008. Acúmulo de macronutrientes por duas cultivares de cebola produzidas em sistema de semeadura direta. Horticultura Brasileira 25: 53-59.

PÔRTO, DRQ; CECÍLIO FILHO, AB; MAY, A; BARBOSA, JC. 2006. Acúmulo de macronutrientes pela cultivar de cebola "Optima" estabelecida por semeadura direta. Horticultura Brasileira 24: 470-475.

PÔRTO, DRQ; CECÍLIO FILHO, AB; MAY, A; VARGAS, PF. 2007. Acúmulo de macronutrientes pela cultivar de cebola "Superex" estabelecida por semeadura direta. Ciência Rural 37: 949-955.

SANGOI, L; ERNANI, PR; LECH, VA; RAMPAZZO, C. 2003. Lixiviação de nitrogênio afetada pela forma de aplicação de uréia e manejo dos restos culturais de aveia em dois solos com texturas contrastantes. Ciência Rural 33: 65-70.

SANTOS, EEF. 2007. Acúmulo de nutrientes e relação Ca:Mg no cultivo da cebola, no submédio São Francisco. Botucatu: UNESP. 120 p. (Tese doutorado).
TAIZ, L; ZEIGER, E. 2009. Fisiologia vegetal. 4. ed. Porto Alegre: Artmed. 819 p.

TEDESCO, MJ; GIANELLO, C; BISSANI, CA; BOHNEN, H. 1995. Análise de solo, plantas e outros materiais. 2.ed. Porto Alegre: UFRGS. 174p. (Boletim Técnico, 5).

VILLAS BÔAS, RL; ANTUNES, CL; BOARETTO, AE; SOUSA, VF; DUENHAS, LH. 2001. Perfil da pesquisa e emprego da fertirrigação no Brasil. In: FOLEGATTI, MV (coord). Fertirrigação: flores, frutas e hortaliças. Guaíba: Agropecuária p. 71-103.

VIDIGAL, SM; PEREIRA, PRG; PACHECO, DD 2002. Nutrição mineral e adubação de cebola. Informe Agropecuário 23: 36-50.

VIDIGAL, SM; PEREIRA, PRG; PACHECO, DD; FACION, CE. 2003. Acumulação de matéria fresca e seca pela cebola. In: CONGRESSO BRASILEIRO DE OLERICULTURA, 43. Resumos... Recife: SOB (CD-ROM).

VIDIGAL, SM; MOREIRA, MA; PEREIRA, PRG. 2010. Crescimento e absorção de nutrientes pela planta de cebola cultivada no verão por semeadura direta e por transplantio de mudas. Bioscience Journal 26: 59-70.

WIEDENFELD, R. 1994. Nitrogen rate and timming effects on onion growth and nutrient uptake in a subtropical climate. Subtropical Plant Science 46: 32-37. 